\title{
EXPERIMENTAL MODAL IDENTIFICATION AND FEM UPDATING OF A SEVEN STORY ISOLATED EDUCATIONAL BUILDING
}

\author{
Rony Reategui ${ }^{1}$, Estefanía Bossus ${ }^{1}$, Diego Villagómez ${ }^{1}$, Mauricio Gonzales ${ }^{1}$, Rafael Aguilar ${ }^{1 *}$ \\ ${ }^{1}$ Engineering Department, Pontifical Catholic University of Peru - PUCP, Lima, Peru
}

Received: 29/06/2019 Accepted: 07/08/2019

\begin{abstract}
The construction of isolated structures is increasing in recent decades in seismic countries. In Peru, the national regulation indicates that important buildings such as hospitals located in areas of high seismic risk must incorporate isolation systems to reduce structural and nonstructural loss. These systems protect the main structure from the effects of a seismic event by separating its base from the earth movement and by reducing the relative displacements and accelerations between adjacent stories. In the structural design process of buildings and seismic protection systems, having numerical models that properly represent the real behavior of the buildings is of high importance. In this context, experimental modal tests represents an attractive cost-effective non-destructive tool to obtain an accurate characterization of the experimental structural response. This paper presents the experimental tests carried out in a base-isolated educational building built in 2014 that has seven stories and three basements with a total built area of around $7500 \mathrm{m2}$. Data acquisition was accomplished with autonomous units (acquisition system and transducers incorporated in a single unit) whose versatility allowed measuring a significant number of degrees of freedom in a limited amount of time. The dynamic properties experimentally identified were used to calibrate the finite element model of the building. The results showed that the design model approximates correctly to the experimentally identified ambient vibration response when considering rigid supporting conditions as well as the interaction of partitioning elements such as walls and parapets.
\end{abstract}

Keywords: Ambient vibration tests, Modal characteristics, Base-isolated building, FEM updating

\section{INTRODUCTION}

The implementation of seismic isolation systems in civil structures is increasingly common in Peru. These systems protect the structure from the effects of an earthquake by separating its base from the movement of the ground with which the drifts and accelerations are reduced. Such is the improvement obtained in terms of seismic performance that the national building regulation already requires its use in strategic buildings, such as important hospitals located in areas of high seismic risk. Due to the importance of these systems, understanding and correctly representing the real behaviour of an isolated structure has become a topic of greater interest.

There were two objective in this research. The first one was to perform the modal identification of a baseisolated educational building, and the second one was to update its numerical model with the modal parameters identified. To achieve these goals, the natural frequencies, damping ratios, and mode shapes were obtained by performing experimental tests using ambient vibrations. The calibration process was performed by assuming certain assumptions about the supporting conditions and rigidity contribution of nonstructural elements.

\section{DESCRIPTION OF THE BUILDING}

Built in 2014, the building is a seven-story baseisolated educational building located at Pontifical Catholic University of Perú in Lima. There are two elevator's boxes and emergency stairs at each end of the structure, and has three levels of basements dedicated to parking. The structural system consists in reinforced concrete moment frames, and has silicacalcareous partition walls between classrooms and in the elevator's boxes and emergency stairs. The floors are mainly waffle concrete slabs, and solid slabs in certain areas. General photos of the building are shown in Figure 1. In addition, it has cantilevered metallic structures located at the central part of the third, fourth, fifth and seventh floor as shown in Figure $1 \mathrm{~b}$. The story heights are $5 \mathrm{~m}$ for first floor and $3.65 \mathrm{~m}$ for second to seventh floors, with a total height of $30.3 \mathrm{~m}$.

\footnotetext{
${ }^{*}$ Corresponding author:

raguilar@pucp.pe
} 


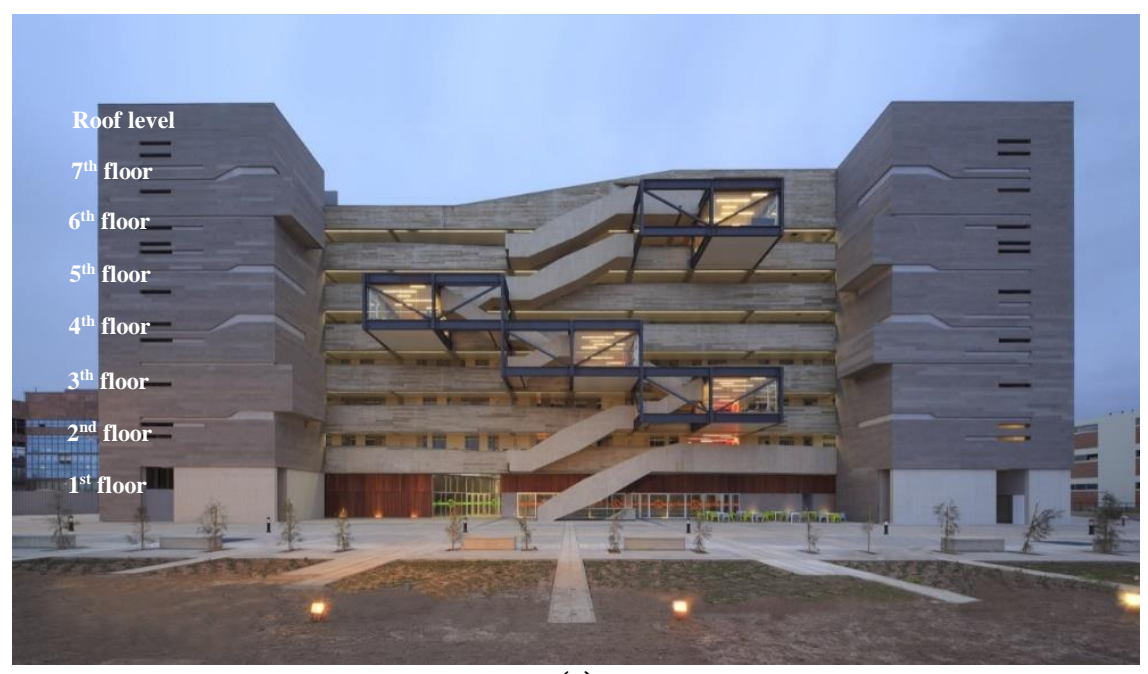

(a)

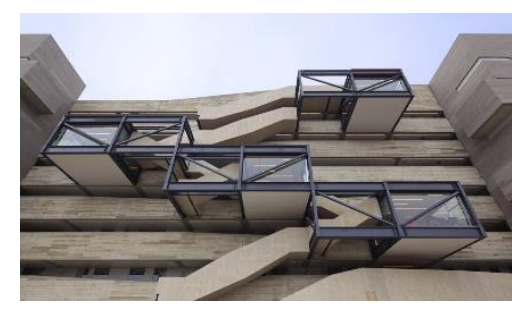

(b)

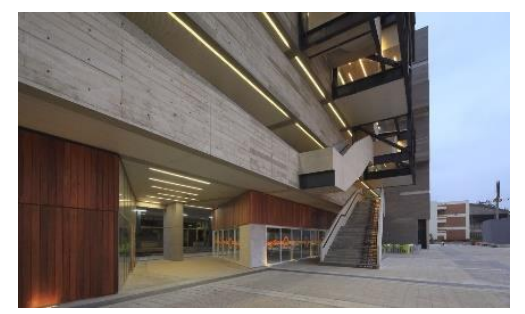

(c)

Figure 1. General photos of the building: (a) front view; (b) cantilevered metallic structures; and (c) principal stairs. (Source: https://www.archdaily.pe/pe/760072/edificio-de-aulas-de-ingenieria-y-ciencias-pucp-llosa-cortegana-arquitectos?ad_medium=gallery)

The three basements reach almost $10 \mathrm{~m}$ deep. The first floor is used for a restaurant and a multi-purpose room. From second to seventh floor there are classrooms. On the roof, there are air conditioning equipment at one side and one central room that is not in use. The typical plan floor of the building is shown in Figure 2.

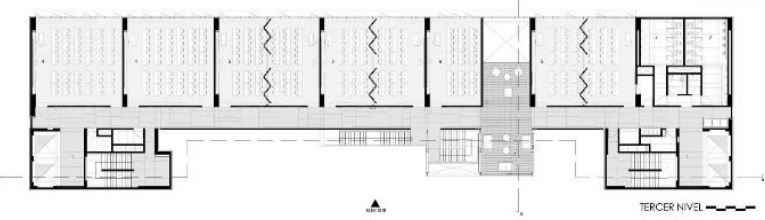

Figure 2. Typical plan floor of the building. (Source: https://www.archdaily.pe/pe/760072/edificio-de-aulas-de-ingenieriay-ciencias-pucp-llosa-cortegana-arquitectos?ad_medium=gallery)

The isolation system is mainly located at the top of the first basement. This system have three types of isolators: two elastomeric isolators (ISO1, ISO2) and one slider isolator (SLD1). The superstructure is supported on 22 base isolators type ISO2 and 3 type SLD1 at level $0.00 \mathrm{~m}$, while the elevator's boxes are 'hung' from the superstructure and supported with 4 base isolators type ISO1 each box at foundation level $-12.20 \mathrm{~m}$. A closeup of the isolation levels and the distribution of isolators at the top of the first basement level are shown in Figure 3 and Figure 5 respectively.

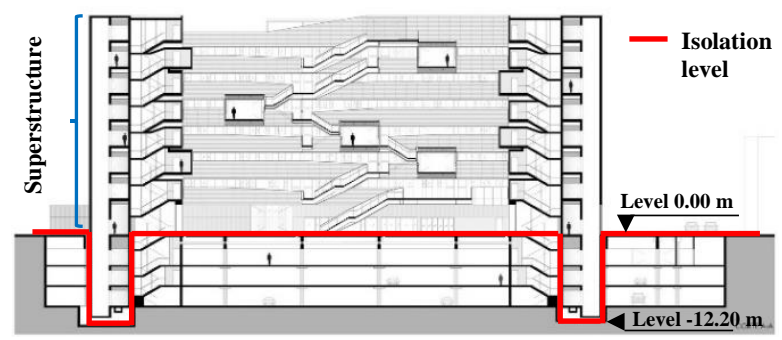

Figure 3. Isolation levels of the structure. (Source: https://www.archdaily.pe/pe/760072/edificio-de-aulas-de-ingenieriay-ciencias-pucp-llosa-cortegana-arquitectos?ad_medium=gallery)

\section{EXPERIMENTAL TESTS}

The experimental program consisted on carrying out ambient vibration tests at selected locations throughout the building. The modal identification was performed with two estimation methods: EFFD ¡Error! No se encuentra el origen de la referencia. and SSI-UPC [2]. This section presents the design of the tests, the analysis of data recorded and the results obtained in the experimental program.

\subsection{Experimental test design}

The measuring equipment were three tri-axial forced balanced accelerometers with data acquisition system (DAQ) included and GPS synchronization, model ETNA2 [3]. These accelerometers have the advantage of being autonomous due to its incorporated DAQ system and the possibility of being used with batteries. In this way, the location of the equipment at the measurement points was faster than the conventional wired system. Figure 4 shows the accelerometer used in the experimental tests. 


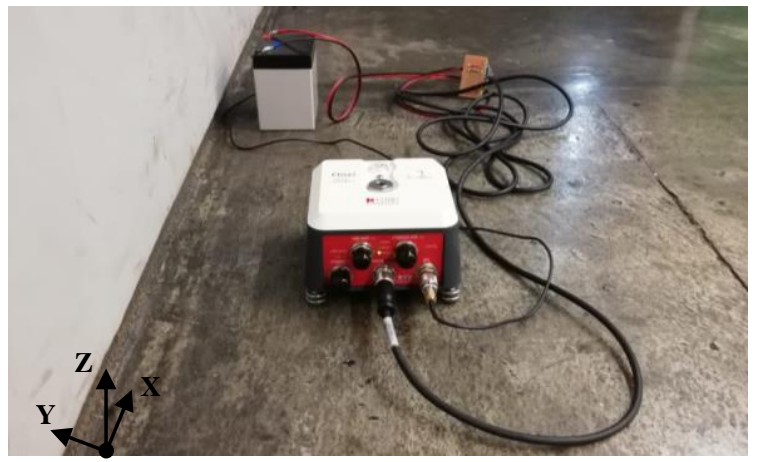

Each testing setup consisted in three accelerometers per floor, located at three corners of the building. A total of four separate setups were used to characterize the dynamic behavior of the structure, measuring on the roof, the sixth and fourth floor levels.

Figure 4. Accelerometer ETNA2 used in the tests.

Isolators at the top of the first basement level $(0.00 \mathrm{~m})$

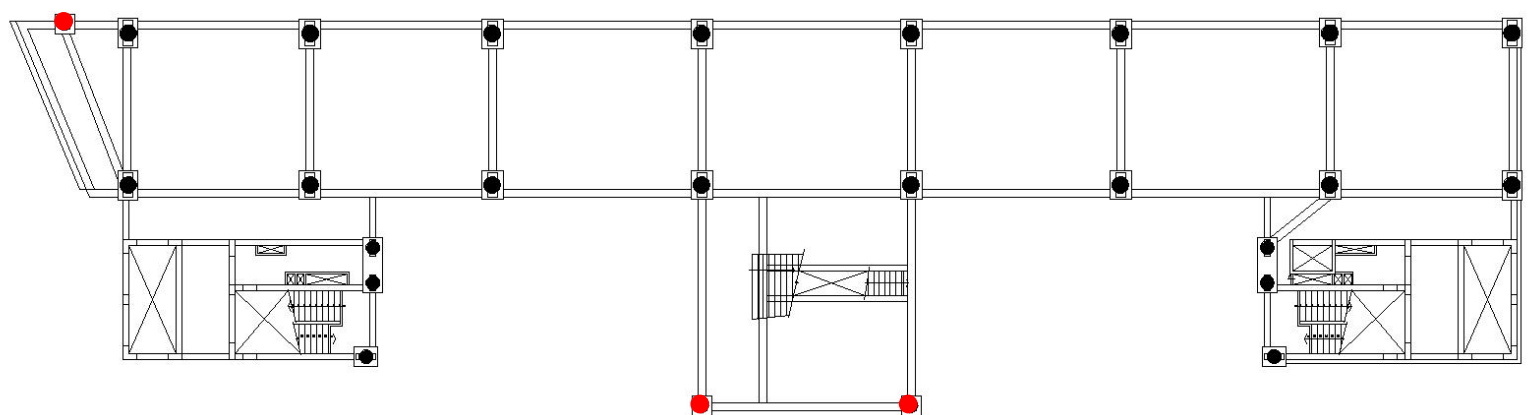

Isolators at the base of the third basement level $(-12.20 \mathrm{~m})$

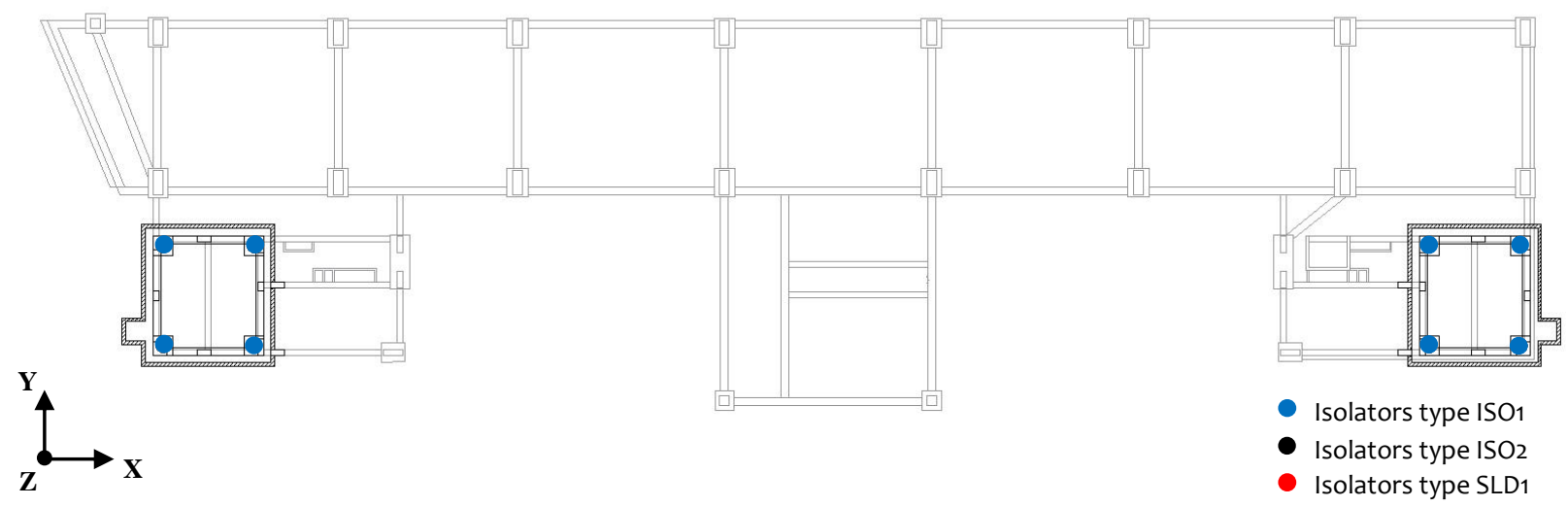

Figure 5. Distribution of the isolators at the isolation levels.

For each setup, a sample time of 25 minutes was adopted to record the data at a sampling rate of $200 \mathrm{~Hz}$. The locations of the accelerometers are shown in Figure 6 , where the local axes of the accelerometers were the same as the global axes of the structure.

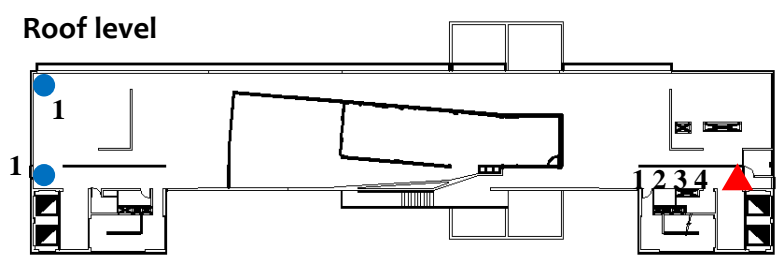

$6^{\text {th }}$ floor
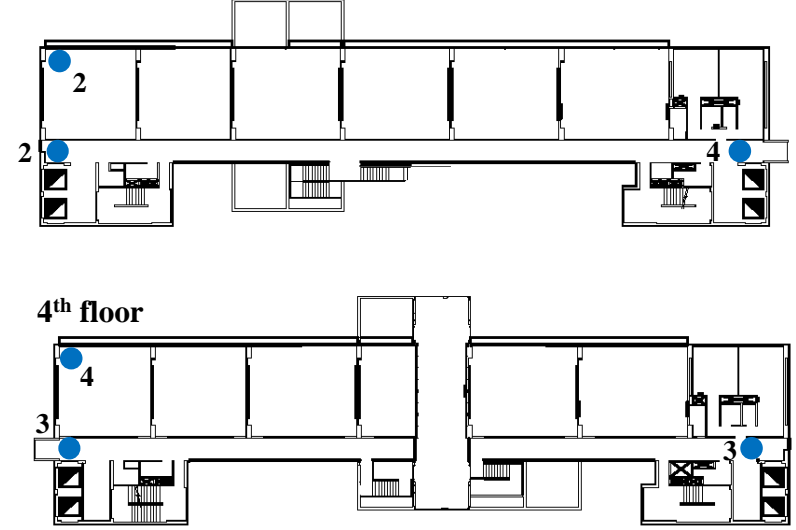

- Reference accelerometer

- Rover accelerometer

1234 Setup

Figure 6. Location of the accelerometers at the building. 


\subsection{Analysis of data recorded}

The experimental tests carried out allowed obtaining modal parameters from the vibrational response to ambient excitations (e.g. wind, traffic, people transit, etc.) and loads in the operational periods of a structure.

The process of record data assume that the ambient vibrations are signals with white noise characteristics, which manage to excite the entire range of frequencies of interest of the structure. Thus, the modal parameters of the structure are identified only evaluating the frequencies with significant strong response [4]. However, the signal also contains noise and some disturbances that may drive the structure at a frequency of unwanted sources (e.g. rotary machines). The modal shape associated to this unwanted frequency is called operational mode. Therefore, the methods used to process the signals must be able to distinguish between natural an operational modes.

In order to cross-validate the results, parametric and non-parametric methods were used to process the data. In the first group, we have the Stochastic Subspace Identification-Unweighted Principal Components (SSI-UPC) method that develops in the time domain. This method fit the vibration data in a parametric model. With this model, it is possible to obtain the frequencies, damping ratios and mode shapes by its transfer function. In the second group, the Frequency Domain Decomposition (FDD) method is presented as an easily processing method to obtain the modal parameters. It is developed in the frequency domain, and consists in a decomposition of the vibration data into a set of responses of single degree of freedom (SDOF) systems. With this, a set of singular values of the spectral density matrix and associated singular vectors are obtained, which represents the natural frequencies and mode shapes respectively. A better method to use is the Enhanced Frequency Domain Decomposition (EFDD). This method is an improvement of the FDD method, which allows to estimate also the damping ratios of the response. For more information about the processing methods see [4].

The main disadvantage of non-parametric methods is that their results depend on the quality of ambient noise. Therefore, parametric methods are usually used more reliably due to the robust numerical algorithms used to perform signal processing [5] and also for its ability of correctly identify structural modes.

In this work, the SSI-UPC and EFDD methods were implemented. They are presented in a Power Spectral Density (PSD) chart for the EFDD method and in a
Stabilization Diagram for the SSI-UPC method. The modal identification process was carried out using the ARTeMIS Modal Pro V5.1 software [6].

\subsection{Experimental Results}

Figure 7 shows the graphs in the use of the EFDD and SSI-UPC methods. Modes obtained with both methods can be correlated using the Modal Assurance Criterion (MAC) [7]. Table 1 shows the values of the fundamental frequencies estimated by both methods, the MAC coefficients and the frequency errors calculated as the difference between the SSI-UPC and EFDD methods, taking the former as reference.

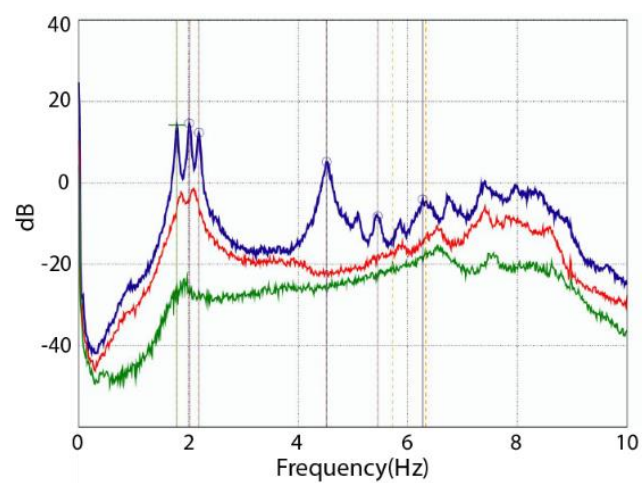

(a)

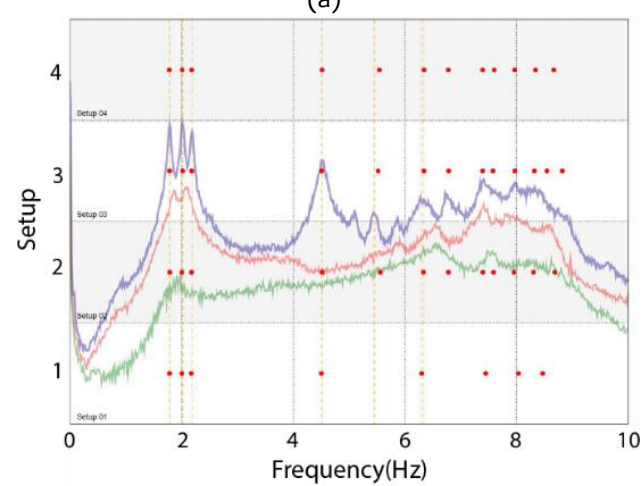

(b)

Figure 7. Experimental results: (a) spectral density chart obtained with EFDD method; and (b) stabilization diagram obtained with SSIUPC method.

The first six modes were selected since they have a MAC coefficient greater than 0.75 , which indicates a good correlation. In Figure $7 \mathrm{~b}$, it can be seen that for setup 1 the fifth frequency could not be detected, thus resulting in a line that deviates at its last point. This explains the MAC value of 0.75 for the corresponding mode and the $3.41 \%$ error between both estimated frequencies. 
Table 1. Fundamental frequencies of the building.

\begin{tabular}{lcccc}
\hline \multirow{2}{*}{ Mode } & \multicolumn{2}{c}{ Frequency $(\mathrm{Hz})$} & \multirow{2}{*}{$\begin{array}{c}\text { Error } \\
(\%)\end{array}$} & MAC \\
\cline { 2 - 3 } & EFDD & SSI & 0.01 & 0.997 \\
\hline 1 & 1.79 & 1.78 & 0.00 & 0.998 \\
2 & 2.02 & 2.01 & 0.00 & 0.996 \\
3 & 2.19 & 2.18 & 0.00 & 0.999 \\
4 & 4.52 & 4.51 & 0.00 & 0.756 \\
5 & 5.46 & 5.55 & 0.02 & 0.00 \\
6 & 6.30 & 6.32 & 0.00 & 0.981 \\
\hline
\end{tabular}

Well-defined vibration modes were found, which are shown in Figure 8. The first mode consisting of a transverse movement can be clearly identified, the second and third are longitudinal modes with a turn at the ends of the building. The fourth is a torsion mode. Finally, the two remaining modes present a more complex behavior. The fifth mode presents a wave motion in the transverse axis and the last mode is a longitudinal movement with a change of direction along its height.

Another important parameter obtained was the damping ratio. This parameter is an indicator of how well the structure dissipates the energy induced by external forces. In case of isolated structures, the isolators are passive devices whose damping ratios

depends on the lateral force applied. Therefore, when performing the modal identification of an isolated structure by means of environmental vibrations, small values of damping are obtained due to the low accelerations induced by the source of excitement [8].

In this way, Table 2 shows the damping values obtained for each mode using the EFDD and SSI-UPC methods. These values turn out to be less reliable than those of the frequencies and mode shapes, since they present large errors which were expected due to the uncertainty and difficulty to correctly identify this parameter through the use of these methods. Even so, all these values vary in the range of 0.5 to $2.5 \%$ and it can be concluded that the structure has small damping ratios according to expectations.

Table 2. Damping ratios of the building.

\begin{tabular}{lccr}
\hline \multirow{2}{*}{ Mode } & \multicolumn{2}{c}{ Damping (\%) } & \multirow{2}{*}{ Error (\%) } \\
\cline { 2 - 3 } & EFDD & SSI & \\
\hline 1 & 1.18 & 0.95 & 24.21 \\
2 & 1.17 & 0.83 & 40.96 \\
3 & 1.21 & 1.17 & 3.42 \\
4 & 1.35 & 1.33 & 1.50 \\
5 & 0.63 & 1.41 & 55.32 \\
6 & 1.11 & 2.19 & 49.32 \\
\hline
\end{tabular}
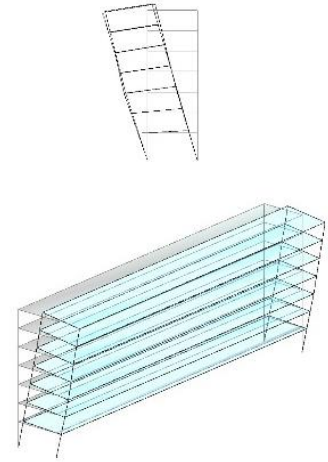

$f=1.78 \mathrm{~Hz}$
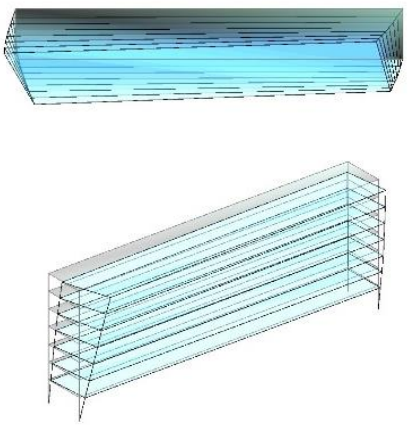

$f=4.51 \mathrm{~Hz}$
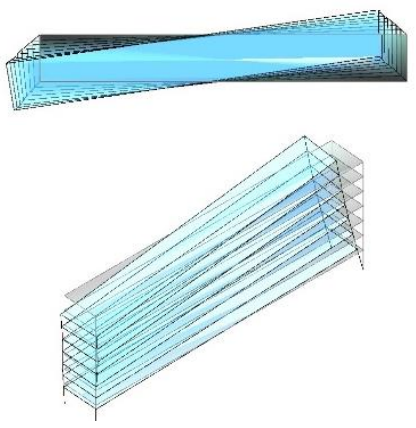

$f=2.01 \mathrm{~Hz}$
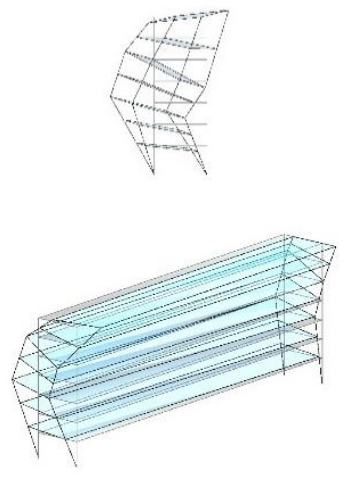

$f=5.55 \mathrm{~Hz}$
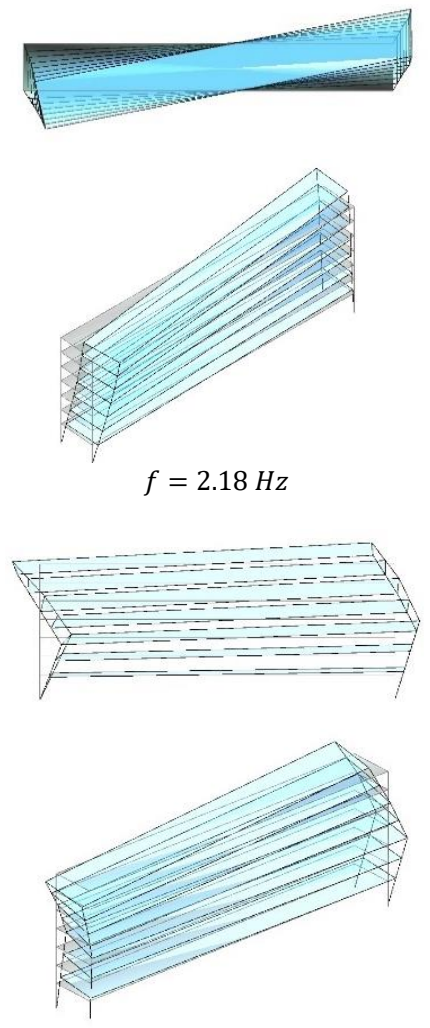

$f=6.32 \mathrm{~Hz}$

Figure 8. First six mode shapes of the building obtained with SSI-UPC method. 


\section{NUMERICAL MODELING AND FEM UPDATING}

The finite element model of the building was developed with the computer program ETABS Version 16.2.1 [9]. The columns, beams, walls and slabs were idealized as an assemblage of area and lines objects. Ritz-vector analysis was adopted to evaluate the fundamental frequencies, mode shapes, modal participation factors, direction factors and participation mass percentages of the structure [10]. The specified compressive strength of concrete used in the model for the concrete elements were 210,280 and $350 \mathrm{~kg} / \mathrm{cm}^{2}$, according to the location and importance of the element. Grade 60 reinforcing steel was also used in the model.

The partition wall loads varied from floor to floor, using a typical value of $0.73 \mathrm{Tn} / \mathrm{m}$ for silica-calcareous ones. The building masses were assumed to be at the floor level, and floor diaphragms rigid in plane. For the modal analysis, $100 \%$ of dead loads and $25 \%$ of live loads were considered.

Other important assumptions were made in order to represent an adequate behavior of the model and obtain reliable results:

-The base was assumed fixed due to the foundation structures.

-Walls and solid slabs were modelled as shell elements, in order to consider its rigidity.

-The base isolators were considered as link elements with two translational and one vertical rigidities.

-The beam-to-beam and beam-to-column connections were considered with rigid offsets. -The whole structure, including the basements and the perimeter walls, was modeled.

Figure 9 presents the finite element model previous to the calibration.

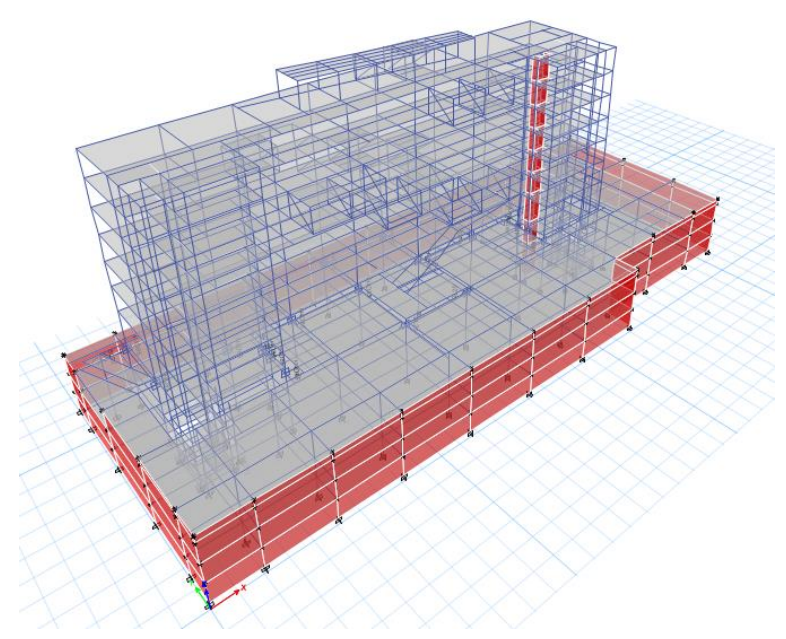

Figure 9. Initial finite element model of the building.

In order to achieve a good correlation between the mode shapes and frequency values determined experimentally and analytically, it was necessary to consider as structural elements the partitions walls used in the classrooms, bathrooms, corridors, elevator's boxes and emergency stairs.

As mentioned, there are three types of base isolators used in this building. However, only the elastomeric ones (ISO1 and ISO2) were which mainly gave its characteristic behavior to the building because they were the majority. Their lateral design stiffness $\left(\mathrm{K}_{2}\right)$, which is used for seismic analysis, had a value of $0.167 \mathrm{Ton} / \mathrm{mm}$ for isolator type ISO1 and $0.131 \mathrm{Ton} / \mathrm{mm}$ for $\mathrm{ISO}_{2}$.

It is known that under ambient vibration conditions, in which the tests were performed, the elastomeric base isolators have a more rigid lateral stiffness $\left(K_{1}\right)$. In this way, the initial values of $K_{2}$ were multiplied by 10, 100, 1000, 10000 times and a fixed case, so that an adequate calibration process of the finite element model is observed.

A graphic shown in Figure 10 was made in order to observe how the frequencies errors vary as the lateral stiffness of the isolators is increased. The error was calculated as the difference respect to the experimental values obtained with SSI-UPC method. It is noticed that for high stiffness's amplification values, the frequency errors are getting smaller. 


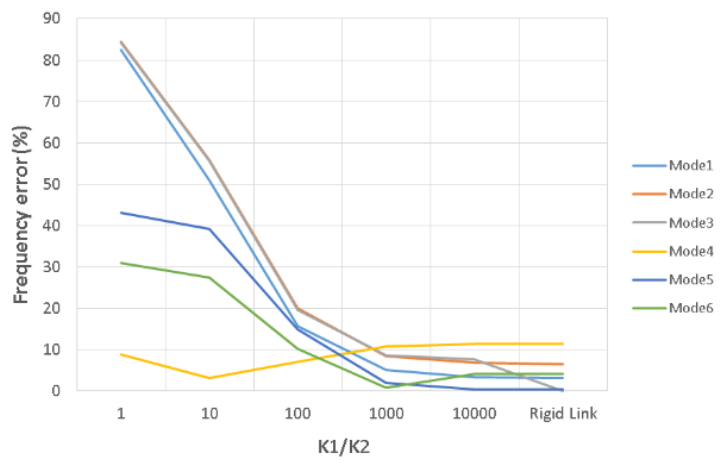

Figure 10. Frequency error values vs. variation of the lateral stiffness of the isolators for all the modes.

The same process was carried out for the mode shapes of the building. MAC values were calculated according to the variation of the lateral stiffness of the isolators. Results are shown in Figure 11.

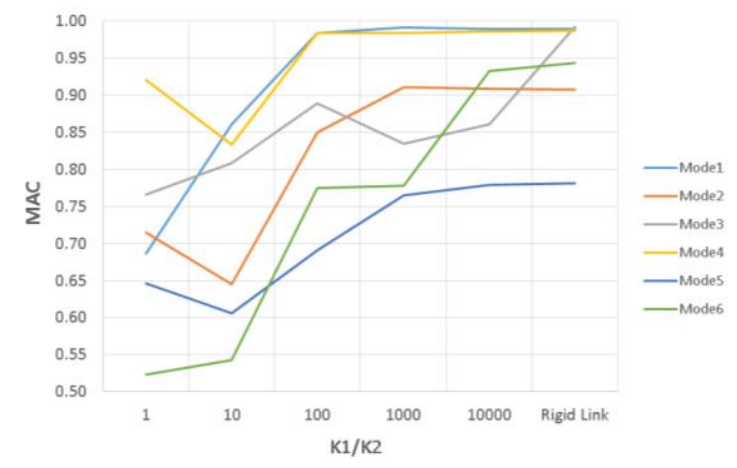

Figure 11. MAC values vs. variation of the lateral stiffness of the isolators for all the modes.

Figure 11 shows that for high stiffness's amplification values, the MAC values are higher, which indicates that the experimental and analytical modal shapes are fitting better. The results obtained show that for very low excitations, the elastomeric isolators behaved like rigid links. This is an interesting result, because it is known that elastomeric isolators has an initial rigidity $\left(K_{1}\right)$ of around 7 to 15 times its design rigidity $\left(\mathrm{K}_{2}\right)$ for low values of displacements.

The final ETABS model of the building is presented in Figure 12, and, as it is observed, the partition walls were modeled (red elements). They gave the structure their characteristic mode shapes to get close to the experimental ones.

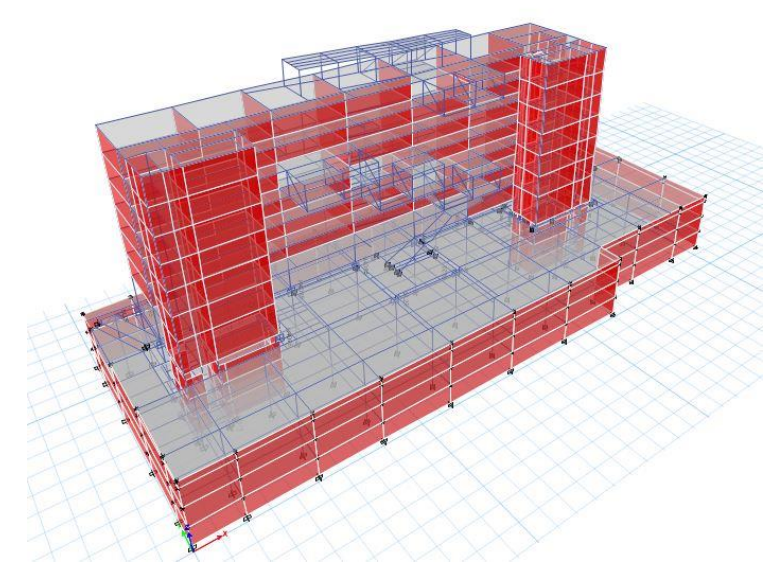

Figure 12. Calibrated finite element model of the building.

The final calibration process ended up in the modal parameters presented in Table 3. Only the frequency and MAC values are presented. It is observed that with both estimation methods and for all modes except fifth, the MAC values are close to 1 . As it was said, the fifth mode couldn't be identified in the first setup. This entail to a non-stable mode, and with this, a distorted mode shape associated to that frequency. That explains the MAC value of 0.423 .

Table 3. Final calibrated values for all the modes of the building.

\begin{tabular}{lccccc}
\hline \multirow{2}{*}{ Mode } & \multicolumn{3}{c}{ Frequencies $(\mathrm{Hz})$} & \multicolumn{2}{c}{ MAC } \\
\cline { 2 - 6 } & EFDD & SSI & ETABS & EFDD & SSI \\
\hline 1 & 1.79 & 1.78 & 1.72 & 0.989 & 0.993 \\
2 & 2.02 & 2.01 & 1.88 & 0.907 & 0.922 \\
3 & 2.19 & 2.18 & 2.18 & 0.992 & 0.990 \\
4 & 4.52 & 4.51 & 5.03 & 0.987 & 0.987 \\
5 & 5.46 & 5.55 & 5.43 & 0.781 & 0.423 \\
6 & 6.30 & 6.32 & 6.58 & 0.943 & 0.942 \\
\hline
\end{tabular}

The natural frequencies and mode shapes of the experimental and analytical model shows accurate results of the calibration process. However, another factors like elasticity modulus of materials, different distribution of the partition walls or even floor eccentricities due to different distribution of live loads could vary considerately the calibration of the model and make it even better. The results presented were obtained with limited information about the material properties, so it is opened to be improved by further investigations.

\section{CONCLUSIONS}

In the present study, the frequencies, damping ratios and mode shapes of a seven-story base-isolated educational building located in Lima, Peru, were experimentally determined. To perform the modal identification, ambient vibrations were taken as source of excitation. The tests carried out consisted of 4 separated setups in which three tri-axial accelerometers with DAQ system incorporated were 
placed at floors $4^{\text {th }}, 6^{\text {th }}$ and roof level. Two estimation methods were used to find the dynamic characteristics of the structure in order to cross-validate the results: EFDD and SSI-UPC.

The results obtained with both estimation methods showed clearly separated fundamental modes, as well as small experimental damping values as expected.

The values obtained experimentally were used to calibrate the finite element model of the structure. It was necessary to consider the contribution of rigidity of non-structural elements such as partition walls between classrooms and in emergency stairs and elevator's boxes. Also, a process of increasing the lateral stiffness of the elastomeric isolators was carried out in order to evaluate its performance against ambient vibrations. Results shown that the isolators had to be assumed as rigid links in case of ambient vibrations. With this, mode shapes managed to look more like the experimentally measured ones and fundamental frequencies were considerably increased, approaching to the experimental values.

The fact that the partition walls are join in with the concrete frames is an issue for further studies due to it is not possible to determine if it is going to affect the structure's behavior with higher levels of vibration such as a seismic event.

\section{ACKNOWLEDGEMENT}

An especial gratefulness to the Engineering \& Heritage laboratory of the Pontifical Catholic University of Peru for the support with the measuring equipment and electronic devices with the necessary software's licenses. The finite element model and plans of the building provided by Prisma Ingenieros was crucial for the success of this investigation too.

\section{REFERENCES}

[1] Brincker R, Ventura C E and Andersen P 2001 Damping Estimation by Frequency Domain Decomposition Proceedings of the $19^{\text {th }}$ international modal analysis conference (IMAC) (Kissimmee, USA)

[2] Structural Vibration Solutions ApS 2001 'Stochastic Subspace Identification' ARTEMIS Modal Help http://www.svibs.com. 17 Jun 2019

[3] Kinemetrics 2017 ETNA2 User's manual https://kinemetrics.com/ 18 Jun 2019

[4] Rainieri C and Fabbrocino G 2014 Operational Modal Analysis of Civil Engineering Structures Springer: New York

[5] Ramos L F, Marques L, Lourenco P B, De Roeck G, Campos-Costa $A$ and Roque $J 2010$ Monitoring historical masonry structures with operational modal analysis: two case studies Mechanical Systems and Signal Processing 24(5) pp 1291-1305

[6] Structural Vibration Solutions ApS 2001 ARTEMIS Modal Pro Release 5.1 User's Manual http://www.svibs.com/ 18 Jun 2019

[7] Allemang R J and Brown D L 1982 A correlation coefficient for modal vector analysis Proceedings of the $1^{\text {th }}$ International Modal Analysis Conference (IMAC) (Orlando, USA)

[8] Ventura C E, Finn W L, Lord J F and Fujita N 2003 Dynamic characteristics of a base isolated building from ambient vibration measurements and low level earthquake shaking Soil Dynamics and Earthquake Engineering 23(4) pp 313-322

[9] Computers and Structures Inc (CSI) 2016 ETABS User's manual http://www.csiberkeley.com 20 Jun 2019

[10] Chopra A K 2001 Dynamics of Structures: theory and applications to earthquake engineering 2 Prentice-Hall: New Jersey 